Website: http://revistas.lamolina.edu.pe/index.php/acu/index

(C) Universidad Nacional Agraria La Molina, Lima - Perú

\title{
Uso de semilla despigmentada de achiote (Bixa Orellana) en el engorde de terneros holstein en crianza intensiva y efecto en el color de la carne
}

\author{
Use of depigmented seed of achiote (Bixa Orellana) in the fattening of holstein calves in intensive \\ aging and effect in the color of the meat
}

\author{
Elzer Pusma Huaman ${ }^{1}$, Pedro Antonio Del Carpio Ramos ${ }^{2}$, Erickson Alvaro Ruiz Figueroa ${ }^{3 *}$ \\ * Autor de correspondencia
}

\section{Resumen}

En la Unidad Experimental de Zootecnia de la Universidad Nacional Agraria La Molina se llevó a cabo un trabajo de investigación con el objetivo de evaluar índices productivos (incremento de peso, consumo de alimento, alzada a la cruz, peso y rendimiento de carcasa y conversión alimenticia), así como mérito económico y coloración de la carne, al incorporar semilla despigmentada de achiote (Bixa orellana) en la dieta de terneros Holstein de cuatro meses de edad. Se utilizaron 14 animales de 4 meses de edad con $135 \mathrm{~kg}$ de peso vivo promedio, estableciéndose dos tratamientos, To: ración testigo y T1: ración con $10 \%$ de semilla despigmentada. Los terneros fueron asignados aleatoriamente a cada uno de los tratamientos, siendo alimentados durante 98 días, para luego ser beneficiados, pesándose las carcasas y tomando muestras para la evaluación del color. Se evaluó estadísticamente mediante un Diseño Completamente Azarizado. Se obtuvieron los siguientes resultados: 5,79 y $5,59 \mathrm{~kg}$ de materia seca consumida por animal por día; 0,99 y $1,04 \mathrm{~kg}$ de incremento de peso vivo por animal-día; 13,7 y $15,0 \mathrm{~cm}$ de incremento de alzada; 125,75 y $129,50 \mathrm{~kg}$ de peso de carcasa; 47,44 y 48,20\% de rendimiento de carcasa; 5,88 y 5,40 de conversión alimenticia; y un mérito económico de 4,20 y 3,62 para cada uno de los tratamientos, respectivamente. Aun cuando las diferencias en consumo de alimento, incremento de peso vivo, alzada, peso y rendimiento de carcasa no tuvieron diferencia estadística significativa, los resultados favorecieron al tratamiento con semilla despigmentada de achiote. Los resultados en conversión alimenticia y mérito económico favorecieron al tratamiento con achiote en 8,3 y 133,8 \%, respectivamente. La coloración de la carne se evaluó mediante el sistema CIELab, y aunque presentó mayores valores el tratamiento con semilla de achiote, no hubo diferencia estadística significativa $(\mathrm{P} \leq 0,005)$, lo cual demuestra que al nivel de $10 \%$ en la dieta durante 14 semanas no afecta el color de la carne.

Palabras clave: crecimiento; terneros; achiote; carne; color.

\begin{abstract}
At the Experimental Unit of Zootecnia of the National Agrarian University La Molina was carried aut a reseach with the objective of evaluating productive indices (increase in weight, food consumption, height at the withers, weight and carcass yield, feed conversion), as well as economic merit and flesh coloring, by incorporating aphigote seed of annatto (Bixa orellana) in the diet of four-month-old Holstein calves. It was used 14 animals of 4 months of age with $135 \mathrm{~kg}$ average, establishing two treatments, To: control ration and T1: ration with $10 \%$ of depigmented seed. The calves were assigned randomly to each of the treatments, being fed for 98 days to then be benefited, weighing the carcasses and taking samples for a color evaluation. It was statistically evaluated by a Completely Randomized Design. The obtained results were: 5,79 and 5,9 kg of dry matter consumed per animal per day; 0,99 and 1,04 kg of increased live weight per animal-day; 13,7 and 15,0 cm increased in height; 125,75 and 129,50 kg of carcass weight; 47,44 and 48,20\% carcass performance; 5,88 and 5,40 of feed conversion; 4,20 and 3,62 of economic merit for each of the treatments, respectively. Even though the differences in feed intake, live weight increase, height, weight and carcass yield did not have a significant statistical difference, the results favored the treatment with annatto seed of achiote. The results in nutritional conversion and economic merit favored the treatment with achiote in 8,3 and $133,8 \%$, respectively. The coloration of the meat was evaluated using the CIELab system, and although there were higher values for annatto seed treatment, they did not have significant statistical difference $(\mathrm{P} \leq 0,005)$, which shows that at the level of $10 \%$ in the diet does not affect the color of meat.
\end{abstract}

Keywords: growth; calves; achiote; meat; color.

\footnotetext{
${ }^{1}$ Egresado de la Escuela Profesional de Ingenieria Zootecnia, Facultad de Ingeniería Zootecnia, Universidad Nacional Pedro Ruiz Gallo, Código postal 14004 - Lambayeque, Perú. Email: elzer04_85@hotmail.com

${ }^{2}$ Escuela Profesional de Ingenieria Zootecnia, Facultad de Ingeniería Zootecnia, Universidad Nacional Pedro Ruiz Gallo, Código postal $14004-$ Lambayeque, Perú. Email: delcarpiofiz@hotmail.com

${ }_{3}^{3}$ Departamento Académico de Producción Animal, Facultad de Zootecnia, Universidad Nacional Agraria La Molina, Apartado postal 12-056- La

Molina, Lima, Perú. Email: ericksonruizf@lamolina.edu.pe
} 


\section{Introducción}

La producción pecuaria es la actividad que aporta en gran medida a la seguridad alimentaria de la sociedad, siendo la proteína animal de nuestra dieta su principal refrente; aportando además, al desarrollo económico naciona. Por ello, se debe tomar en consideración que en el engorde de bovinos, la alimentación representa el $70 \%$ del total de costos de producción, por lo que para tener una mejor rentabilidad, se deben buscar alternativas que mejoren el nivel nutricional y por ende los costos. Hidalgo (2014) menciona que todas las proteínas de origen animal y vegetal son bien aprovechadas por los rumiantes, siendo estos animales capaces de sintetizar proteína en el rumen a partir de fuentes simples de nitrógeno, por acción de la microflora bacterial (bacterias y protozoarios) que fabrica proteína bacterial aprovechable por el animal, por lo que es posible preparar raciones sencillas con solo dos o tres ingredientes. Asimismo, en la industria lechera nacional, sobre todo en el sistema intensivo, existe la posibilidad de aumentar la rentabilidad a base de los terneros machos que representan en promedio el $50 \%$ de los nacimientos, los cuales son considerados un subproducto de la industria y vendidos a los pocos días de nacidos para su crecimiento y engorde, siendo baja su demanda y los precios de venta en el mercado. Por ello es que se deben buscar alternativas para revertir esta situación.

El achiote (Bixa orellana) tiene una variedad de denominaciones como Achote, Anato, Bija, Bixa, Urucú, Uñañé, Onoto, Eroyá, Pumacoa (Brack, 1999), es empleado por el alto contenido de pigmentos en la parte envolvente de sus semillas, quedando una cantidad considerable de material que puede ser utilizado en la producción animal; específicamente como insumo alimenticio para animales de interés zootécnico (Banco Agrario, 1988). Cabieses (1993) menciona un valor medicinal del achiote, y luego Silveira et al (2011) demostraron la factibilidad económica de utilizarlo para prevenir las infecciones e infestaciones de las heridas producidas por el marcaje con hierro candente en terneros usando una formulación hidrófila de achiote y sábila (Aloe vera).

Se han realizado diversos ensayos de alimentación con el achiote, habiéndose aplicado en aves de interés zootécnico, específicamente en pollos, gallinas, pavos y patos de carne; evaluándose en un inicio sobre su efecto en la capacidad de pigmentación de piel y huevos. Obando (1990), estableció que era positivo el efecto de la inclusión de la semilla de achiote despigmentada en raciones de vacas lecheras, tanto por su aceptación como por los bajos costos.

El color de la carne fresca es el principal atributo que influye en la decisión de compra, dado que el consumidor asocia el color con el grado de frescura y calidad (Brewer et al., 2002). En la carne, al igual que otros materiales no metálicos, al incidir un rayo de luz en su superficie se produce una reflexión difusa, esa reflexión es lo que se define como el color (Braña et al, 2011). El color percibido ha sido definido por CIE (Commision Internationale de
L'Eclairage) como el atributo visual que se compone de una combinación cualquiera de componentes cromáticos y acromáticos, el cual es una cualidad organoléptica de los alimentos y se aprecia por medio del sentido físico de la vista. También suele ser considerado un factor psicológico de apreciación y un criterio para elegir un producto alimenticio (Mathias-Rettig et al, 2014). En 1976, la CIE propuso una modificación a la escala original (Hunter $\mathrm{L}, \mathrm{a}, \mathrm{b})$ al calcular de forma diferente los valores siendo nombrados: $L^{*}, a^{*}, b^{*}$ (luminosidad, enrojecimiento y amarillamiento respectivamente) lo que ahora se conoce como el espacio de color CIEL*a*b* (Braña et al, 2011). Estos mismos autores mencionan que al realizar la determinación de color en el músculo, el parámetro de $\mathrm{L}^{*}$ se correlaciona con el estado físico de la carne, la estructura de las fibras musculares y la cinética implicada para establecer el rigor mortis; mientras que el tono es determinado por el estado químico del pigmento de mayor concentración en la carne, la mioglobina $(\mathrm{Mb}$, de color rojo púrpura; oximioglobina, $\mathrm{MbO} 2$, de color rojo vivo; metamioglobina, MetMb, de color pardo), y el croma se relaciona más con la concentración de mioglobina, que influye directamente en la saturación del color del músculo y se relaciona principalmente con los factores ante-mortem (tipo de músculo, edad, alimentación, genética).

El objetivo del presente trabajo fue evaluar los índices productivos (incremento de peso, consumo de alimento, alzada a la cruz, peso, rendimiento de carcasa y conversión alimenticia), así como el mérito económico y color de la carne al incorporar semilla despigmentada de achiote (Bixa orellana) en la dieta de terneros Holstein de cuatro meses de edad para la producción de carne.

\section{Materiales y métodos}

\section{Localización y duración}

El experimento se realizó en la Unidad Experimental de Zootecnia de la Universidad Nacional Agraria La Molina - Lima, teniendo la fase de campo una duración efectiva de catorce semanas (20 de mayo al 26 de agosto del 2008); el beneficio se hizo en el Camal de Conchucos - Lima, y la evaluación de la carne en el Laboratorio de Tecnología de Alimentos de la Facultad de Indusrias Alimentarias de la UNALM.

\section{Tratamientos}

Tratamiento control (To): Testigo, dieta de alimento sin achiote. Tabla 1. Tratamiento 1 (T1): Dieta de alimento con $10 \%$ de semilla despigmentada de achiote, cuya composición se indica enel Tabla 1.

\section{Características del material y equipo experimentales}

Se emplearon catorce terneros Holstein en crecimiento, de 4 meses de edad, con un peso vivo promedio de 135 kilos al inicio del ensayo, empleándose raciones iso-energéticas e iso-proteicas, con un aporte de $65 \%$ de NDT y $14,4 \%$ de proteína cruda. 


\section{$\underline{\text { Producto Evaluado }}$}

Semilla despigmentada de achiote (Bixa orellana) obtenida después de habérsele extraído la capa de pigmento, la que se emplea principalmente para la fabricación de colorantes de uso humano.

\section{Instalaciones y Equipo}

Se emplearon dos corrales de $35 \mathrm{~m}^{2}$ cada uno provistos de comedero, bebedero, zona sombreada, así como dos balanzas tipo plataforma, una de 1 tonelada y otra de 100 kilos de capacidad.

Tabla 1. Composición porcentual de las raciones y valor nutritivo

\begin{tabular}{|c|c|c|}
\hline Ingredientes & Trat. control & Tratamiento 1 \\
\hline Afrecho de trigo & 71,71 & 59,56 \\
\hline Melaza de caña de azúcar & 09,99 & 10,14 \\
\hline Torta de soja & 04,97 & 05,56 \\
\hline Sal común iodada & 00,19 & 00,19 \\
\hline Panca de maíz, molida & 07,39 & 07,11 \\
\hline Maíz amarillo, grano molido & 04,26 & 05,03 \\
\hline Carbonato de calcio & 01,40 & 01,43 \\
\hline Pre-mezcla vitamínico mineral & 00,09 & 00,10 \\
\hline Achiote, semilla despigmentada & 00,00 & 10,90 \\
\hline \multicolumn{3}{|l|}{ Aporte estimado de: } \\
\hline Materia seca, $\%$ & 86,91 & 86,72 \\
\hline NDT, $\%$ & 65,69 & 65,61 \\
\hline Proteína, \% & 14,42 & 14,43 \\
\hline Fibra cruda, $\%$ & 12,30 & 12,13 \\
\hline FDN, \% & 40,43 & 41,88 \\
\hline $\mathrm{FDA}, \%$ & 13,71 & 14,69 \\
\hline ENmantenimiento, Mcal/ kg & 01,56 & 01,56 \\
\hline ENganancia, Mcal/ kg & 00,95 & 00,95 \\
\hline Grasa, \% & 03,18 & 02,97 \\
\hline Calcio, $\%$ & 00,83 & 00,84 \\
\hline Fósforo, \% & 00,79 & 00,74 \\
\hline
\end{tabular}

\section{Técnicas experimentales}

Los animales provenientes del establo fueron desparasitados mediante dosificación con Ivermectina, realizándose un período de acostumbramiento de 10 días, en el que se suministró progresivamente la ración experimental, en reemplazo de chala de maíz, la que se suministró luego en cantidades mínimas. Los animales fueron asignados aleatoriamente a los dos tratamientos, procediéndose a realizar una pesada inicial y posteriormente cada 7 días, hasta completar las 14 semanas experimentales. Durante el experimento el alimento concentrado fue suministrado ad libitum. Finalizada la fase de campo, los animales fueron trasladados y faenados en el Camal de Conchucos. Las canales se colocaron en un cuarto frío con temperatura de $4^{\circ} \mathrm{C}$ por un periodo de $24 \mathrm{~h}$, tiempo después del cual se tomaron una muestras del músculo Longissimus dorsi de $2,5 \mathrm{~cm}$ de espesor y de ellas una porción de $2 \mathrm{~cm}^{2}$, las cuales se colocaron en una bolsa de cierre hermético previamente identificada, llevándose inmediatamente al Laboratorio de
Tecnología de Alimentos de la UNALM para realizar el análisis del color. Se registró el espectro de reflectancia entre 400 y $700 \mathrm{~nm}$ mediante un espectrofotómetro. Las coordenadas de color (Luminosidad, L*; enrojecimiento, $a^{*}$; amarillamiento, $b^{*}$ ) se calcularon en el sistema CIELab.

\section{Variables evaluadas}

Se evaluó el consumo de alimento, peso vivo ( $\mathrm{kg})$, alzada (cm), conversión alimenticia, mérito económico, peso y rendimiento de carcasa y coloración de la carne

\section{$\underline{\text { Análisis estadístico }}$}

Se utilizó la Prueba de Bartlett de homogeneidad de varianzas para verificar la suposición de homocedasticidad (distribución homogénea de la componente residual de varianza) con los pesos corporales iniciales y los incrementos de peso de los animales; y el análisis de varianza para determinar el valor de $\mathrm{F}$ que permita determinar la existencia o no de diferencias significativas entre los tratamientos. Asimismo, se hicieron análisis de covarianza entre peso inicial e incrementos de peso para descartar o corregir el efecto de la variable concomitante sobre los incrementos de peso.

\section{Resultados y discusión}

\section{Consumo de alimento}

Para los tratamientos To (control) y $\mathrm{T} 1$, el consumo promedio de materia seca por animal fue de 568,73 y 549,71 kilos respectivamente; cantidades que, llevadas a base diaria, representaron 5,79 y 5,59 kilos de materia seca por animal. La presencia de $10 \%$ de achiote en el concentrado representó una disminución en el consumo de $3,4 \%$. Una reducción de $4 \%$ en el consumo de alimento puede resultar trascendente para animales tiernos; trascendencia que disminuye conforme los animales van aumentando en edad. En el caso de vacunos de más de 300 kilos de peso corporal puede representar una diferencia intrascendente, sin embargo, tal diferencia debe considerarse en función del rendimiento obtenido por los animales. En no rumiantes, aves y cerdos principalmente, el empleo de achiote y de otras especies vegetales que contienen sustancias parecidas (polifenoles) también ha producido reducciones en el consumo de alimento en determinadas situaciones atribuidas a una mejor utilización del alimento (Bazan, 2005).

\section{Peso vivo}

Los animales de los tratamientos To (control) y T1 tuvieron pesos promedio iniciales de 135,9 y 134,4 kilos respectivamente. Al realizar la prueba de homogeneidad de varianzas de Bartlett, se encontró que la componente residual de varianzas estuvo uniformemente distribuida. En el mismo orden de tratamientos, los pesos finales fueron de 232,60 y 236,43 kilos, promedio por ternero, presentando incrementos de peso promedio de 96,7 y 101,9 kilos para los 
tratamientos control y 1 respectivamente. La componente residual de varianzas estuvo uniformemente distribuida y las diferencias entre los tratamientos no alcanzaron significación estadística aun cuando el incremento de peso promedio del tratamiento 1 superó en 5,4\% al del Tratamiento control, no encontrándose efecto significativo de la variable concomitante Peso inicial. Araúz (2009), menciona que los terneros Holstein de cuatro y ocho meses de edad tienen en promedio 130,60 y 201,40 kilos de peso vivo respectivamente, valores inferiores a los reportados en esta investigación.

Efectos de la desaceleración del aumento de peso vivo podría ser indicativo de efecto acumulativo de toxicidad de ligera intensidad, siendo este tipo de comportamiento reportado para aves de carne con diferentes especies vegetales, con uso continuado y con proporciones mucho menores a las empleadas en el presente trabajo de investigación (Gonzalez, 2009; Lara, 2009). Los incrementos de peso vivo acumulados fueron relativamente mejores en el tratamiento $1(5,4 \%$ en incremento de peso vivo y $9,5 \%$ en incremento de alzada a la cruz), lo que es indicativo de que el achiote ejerce algún tipo de acción nutricional sobre la fisiología de la flora bacteriana. Este efecto podría darse sobre la producción de gases a nivel del rumen, permitiendo que se pierda menos energía en forma de metano y esta se destine para procesos de producción. Tonani et al. (1995) en una evaluación de bovinos en confinamiento reportó un aumento de peso de $0,827 \mathrm{~kg}$ / animal) y la ingesta de materia seca (2,2\% de su peso vivo) cuando fueron alimentados con dietas con niveles de 75 por ciento de concentrado y 35 por ciento de semillas de urucum (achiote).

En un trabajo de investigación desarrollado por Albitres (1992), concluye que hubo un efecto biológico positivo al nivel de 5 por ciento de semilla de achiote parcialmente despigmentado en la alimentación de cerdos en la fase de crecimiento. En aves de carne y cerdos, la presencia de sustancias vegetales (como el achiote) en la dieta han permitido controlar flora patógena (Cozzolino et al., 1998; Fyfe et al., 1998; Williams y Losa, 2001), acción que podría manifestarse en el rumen.

\section{$\underline{\text { Alzada }}$}

La alzada inicial a la cruz fue en promedio de 98,3 y 97,1 $\mathrm{cm}$ respectivamente para los tratamientos control y $1 . \mathrm{La}$ componente residual de varianza estuvo uniformemente distribuida, lográndose una alzada final de 112,0 y $112,1 \mathrm{~cm}$, por lo que los incrementos de alzada fueron respectivamente, de 13,7 y $15.0 \mathrm{~cm}$. Entre los incrementos de alzada la componente residual de varianza estuvo uniformemente distribuida y no se registraron diferencias significativas entre los tratamientos. El análisis de covarianza indicó que después de corregir por efecto del peso inicial, las diferencias entre tratamientos continuaron siendo no significativas aun cuando el tratamiento 1 tuvo incrementos de alzada superiores en 9,5\% a los del Tratamiento control. Los resultados con los indicadores del crecimiento (peso y alzada a la cruz) han puesto de manifiesto que la inclusión de semilla despigmentada de Achiote en el concentrado de los terneros no afectó negativamente el rendimiento en vivo; por el contrario, estos se mejoraron aun cuando estadísticamente no se alcanzó significación estadística. Araúz (2009), menciona que los terneros Holstein de cuatro y ocho meses de edad tienen en promedio 92,0 y $102,0 \mathrm{~cm}$ de altura respectivamente, cuyos valores son inferiores a los encontrados en el presente trabajo de investigación.

\section{Peso y rendimiento de carcasa}

Se beneficiaron los animales obteniéndose pesos de carcasas promedio de 125,75 y 129,5 kilos para los tratamientos control y 1 respectivamente y ambas clasificadas como primera. La prueba de homogeneidad de varianzas permitió determinar que la componente residual de varianza no estuvo uniformemente distribuida, por lo que los pesos de las caracasas fueron llevados a base logarítmica para aplicar el análisis de la varianza, que determinó que las diferencias entre los tratamientos no alcanzaron significación estadística, aun cuando el peso promedio de carcasa del tratamiento 1 (con Achiote) superó en $3 \%$ al del tratamiento testigo. Los rendimientos de carcasa fueron de 47,44 y 48,20\% en el mismo orden de tratamientos, y al aplicar la prueba de homogeneidad de varianzas se determinó que hubo homogeneidad, y que además las diferencias entre tratamientos no fueron significativas. Como en el caso del rendimiento en vivo, las evaluaciones de las carcasas de animales que consumen achiote despigmentado en la proporción de $10 \%$ no desmejoran el rendimiento, aún en animales tiernos.

\section{Conversión alimenticia y mérito económico}

Para los tratamientos control y 1 , los valores de conversión alimenticia fueron 5,881 y 5,395 respectivamente, demostrando ventaja los animales alimentados a base de dieta con achiote, lo cual no evidencia que el consumo continuado de especies vegetales como el achiote, que contienen terpenos, pueden dar lugar a algún efecto acumulativo de toxicidad; más aun si la proporción empleada es de magnitud considerable (Gonzales, 2009; Lara, 2009). Se deduce que el mérito económico en el tratamiento con achiote fue superior en 13,8 \%, cuya base radica en la relativa menor cantidad de alimento consumido, mayor incremento de peso y el menor costo de la semilla despigmentada de achiote.

\section{Coloración de la carne}

En el Tabla 2 se presenta los resultados de coloración de la carne, determinados con el sistema CIELab. Si bien no se registraron diferencias significativas entre los tratamientos para las diferentes coordenadas del color, se aprecia que las carcasas de los animales del tratamiento 1 exhibieron coordenadas ligeramente mayores; indicando que fueron de coloración ligeramente más intensa. La razón principal 
para explicar este resultado radica en que, si bien al Achiote se le ha extraído la mayor parte de la cubierta pigmentada, aun mantiene un remanente de los pigmentos, los que aparentemente superan la acción bacteriana a nivel del rumen y se absorben hasta depositarse en el tejido muscular. El Achiote posee pigmentos de coloración roja y amarilla, predominando los primeros; apreciándose un mayor efecto sobre el enrojecimiento $\left(a^{*}\right)$ que sobre el amarillamiento $\left(b^{*}\right)$. Esto puede representar una ligera ventaja para la comercialización si se tiene en cuenta que en la carne de terneros la coloración roja tiende a ser de mucha menos intensidad y que en algunos consumidores puede no ser muy bien aceptado; así, un ligero incremento en la coloración rojiza podría ser conveniente.

Tabla 2. Coloración de la carne de terneros Holstein sin y con semilla de Achiote (Bixa orellana) despigmentada en la dieta

\begin{tabular}{ccc}
\hline & \multicolumn{2}{c}{ Tratamientos } \\
Momento & Control & 1 \\
\hline Inmediatamente después del sacrificio: & \\
$\mathrm{L}^{*}$ & 38,12 & 38,54 \\
$\mathrm{a}^{*}$ & 12,41 & 13,12 \\
$\mathrm{~b}^{*}$ & 2,68 & 2,14 \\
24 horas posteriores al sacrificio: & \\
$\mathrm{L}^{*}$ & 42,15 & 43,43 \\
$\mathrm{a}^{*}$ & 14,98 & 16,29 \\
$\mathrm{~b}^{*}$ & 5,36 & 5,82 \\
\hline
\end{tabular}

Fernández et al. (1996), trabajaron con terneros Holstein que fueron faenados cuando alcanzaron alrededor de 205 kilos de peso vivo en los que la coloración a los 45 minutos post-mortem fue $\mathrm{L}^{*}$ de 48,3 , a* de $8,3 \mathrm{y} \mathrm{b}^{*}$ de 3,78 ; a las 48 horas post-mortem los valores, en el mismo orden de coordenadas, fueron de 54,4, 12,9 y 10,9. Estos valores son mayores a los encontrados, por Pardomingo et al. (2007), quienes trabajando con terneros Angus, determinaron valores de L*, a* y b* de 35,4, 14,9 y 15,3 , respectivamente; concordantes en luminosidad y enrojecimiento con los resultados obtenidos en el presente experimento, pero fueron de mayor grado de amarillamiento.

\section{Conclusión}

El tratamiento con semilla de achiote a un $10 \%$ en la dieta durante 14 semanas no afecta el color de la carne. $\mathrm{S}$ esugiere realizar estudios con porcentajes mayores para observar posibles cambios.

\section{Literatura citada}

Albitres, M. 1992. Utilización de la semilla de achiote (Bixa orellana) parcialmente despigmentada en la alimentación de cerdos en la fase de crecimiento.
Tesis para optar el título de ingeniero Zootecnista). Universidad Nacional Agraria de la Selva (UNAS). Tingo María, Perú.

Araúz, E. 2009. El registro individual y la tarjeta de producción y reproducción para la valoración de registro en el ganado bovino de leche y carne. Programa académico. Programa de la Asignatura LCPA 445, Departamento de Zootecnia, Facultad de Ciencias Agropecuarias, Universidad de Panamá.

Banco Agrario. 1988. Análisis de rentabilidad del cultivo de achiote. $2^{\mathrm{a}}$ ed. Oficina Central de Promoción y Desarrollo, División de Promoción Empresarial, Departamento de Divulgación. Lima, Perú.

Bazan, M. 2005. Rendimiento de pollos de carne que recibieron fitobióticos en la dieta y bioestimulante en el agua de bebida, sin antibiótico promotor de crecimiento. Tesis. Facultad de Ingeniería Zootecnia, Universidad Nacional "Pedro Ruiz Gallo". Lambayeque, Perú.

Brack, A. 1999. Diccionario Enciclopédico de Plantas Útiles del Perú. Programa de las Naciones Unidas para el Desarrollo; Centro de Estudios Regionales Andinos Bartolomé de Las Casas, Cusco 556p.

Braña, D.; Ramírez, E.; Rubio, M.; Sánchez, A.; Torrescano, G.; Arenas, A.; Partida, J. y Ponce, E.; Ríos, F. 2011. Manual de Análisis de Calidad en Muestras de Carne. Centro Nacional de Investigación Disciplinaria en Fisiología y Mejoramiento Animal Folleto Técnico No. 11 1ra. Ed. Octubre 2011. ISBN: 978-607-425-612-3 Queretaro, Mexico.

Brewer, S.; Wilson, J. and McKeith F. 2002. The effect of pig genetics and palatability, colorant physical characteristics of fresh loin chops. Meat Science 2002; 61: 249-256.

Cabieses, F. 1993. Apuntes de Medicina Tradicional. La racionalización de lo irracional. Concejo Nacional de Ciencia y Tecnología. CONCYTEC. Lima, Perú. 277284 p.

Cozzolino, F.; Furgon-Koiij, J.; Manzini, A.; Torres, C. 1998. Actividad antioxidante del extracto de canela (Cinamomum zeylanicum Breyne). Bioquímica (Sao Paulo, Brasil), 137(11): 437-447.

Fernández, X.; Monin, G.; Culioli, J.; Legrand, I.; Quilichhini, I. 1996. Effect of duration of withdrawal and transportation time on muscle characteristics and quality in Friesian-Holstein calves. J. Anim. Sci., 74: 1576-1583.

Fyfe L.; Smith-Palmer, U. y Stewart, J. 1998. Propiedades antimicrobianas de esencias y de aceites esenciales de plantas contra cinco patógenos importantes en alimentos. Microbiología, 26(2): 118.122.

Gonzales, A. 2009. Acción productiva de infusiones de follaje de saúco (Sambucus nigra) en el pollo de carne. Tesis. Facultad de Ingeniería Zootecnia, Universidad Nacional "Pedro Ruiz Gallo". Lambayeque, Perú.

Hidalgo, V. 2014. Nutrición de rumiantes. Apuntes de 
clase. Facultad de Zootecnia. Universidad Agraria La Molina. Lima, Perú.

Lara, C. 2009. Acción productiva del saúco (Sambucus nigra) en la dieta de pollos de carne. Tesis. Facultad de Ingeniería Zootecnia, Universidad Nacional "Pedro Ruiz Gallo”. Lambayeque, Perú.

Mathias-Rettig, K. y Ah-Hen, K. 2014. El color en los alimentos un criterio de calidad medible Escuela de Graduados, Facultad de Ciencias Agrarias, Universidad Austral de Chile. Agro Sur, 42(2):07-39.

Obando, O. 1990. Efecto de la inclusión de la semilla de achiote (Bixa orellana) despigmentada en raciones de vacas lecheras. (Tesis para optar el título de Ingeniero Zootecnista). Universidad Nacional Agraria La Molina (UNALM). Lima, Perú.

Pardomingo, A.; Volpi, G.; Irurueta, F.; Carduza, P.; Garcia, A. 2007. Característica de la carne de terneros alimentados a corral con maíz entero o molido, metionina y núcleo mineral enriquecido. Rev. Argentina de Prod. Animal., 27(1): 376-377.

Silveira, E.; Benítez R.; Cuesta, M.; Norman, O. 2011. Efectividad de una formulación hidrófila de Bixa orellana L. y Aloe vera L. en el tratamiento de quemaduras en terneros. Revista electrónica de Veterinaria, 12 (1 ): 1695-7504 2011.

Tonani, F.; Alcalde, C.; Ruggieri, A.; Andrade, P.; Guim. A.; Silva, A.; Valadares, S.; Silva, E.; 1995. Utilizaçao da semente de urucum (Bixa orellana, L.) em dietas para bovinos de corte em confinamento. Reuniao Anual da Sociedade Brasileira de Zootecnia. Sociedade Brasileira de Zootecnia (SBZ). 32: 285-286.

Williams, P.; Losa, R. 2001. The use of essential oils and their compounds in poultry nutrition. World Poultry Elsevier, 17(04): 14 - 15. 